\title{
Effect of initiator on photopolymerization of acidic, aqueous dental model adhesives
}

\author{
Xinglin Guo ${ }^{1,2}$, Zhonghua Peng ${ }^{3}$, Paulette Spencer ${ }^{4}$, and Yong Wang ${ }^{1}$ \\ ${ }^{1}$ Department of Oral Biology, University of Missouri-Kansas City School of Dentistry, Kansas City, \\ Missouri 64108 \\ ${ }^{2}$ Institute of Chemistry, Chinese Academy of Sciences, Beijing 100190, China \\ ${ }^{3}$ Department of Chemistry, University of Missouri-Kansas City, Kansas City, Missouri 64110 \\ ${ }^{4}$ Bioengineering Research Center, School of Engineering, University of Kansas, Lawrence, \\ Kansas 66045
}

\begin{abstract}
This study evaluated different initiator systems in self-etching model adhesives, in which camphorquinone (CQ) or [3-(3,4-dimethyl-9-oxo-9H-thioxanthen-2-yloxy)-2-hydroxylpropy] trimethylammonium chloride (QTX) was employed as a photoinitiator (dye). $N$-phenylglycine (NPG), ethyl 4-dimethylaminobenzoate (4E) or 2-(dimethylamino) ethyl methacrylate (DMAEMA) was used as the coinitiator (CI). The role of diphenyliodonium hexafluorophosphate (DPIHP) in the polymerization process was also studied. The concentrations of dye, CI, and DPIHP in model adhesives were all maintained at $0.022 \mathrm{mmol}$ per gram monomer. The model adhesive contained two monomers: (bis[2-(methacryloyloxy)ethyl] phosphate) (2MP) and 2hydroxyethyl methacrylate (HEMA) whose mass ratio was 1:1, thus representing an acidic and hydrophilic formulation. The polymerization rate and the degree of conversion (DC) of the model adhesives with 5,15 , or $25 \%$ water content were determined using FTIR/ATR with a time-based spectrum analysis. The results indicated that with $\mathrm{CQ}$ as the photoinitiator, $4 \mathrm{E}$ appeared to be the most efficient $\mathrm{CI}$, whereas the CQ-DMAEMA combination led to very low radical generation efficiency (DC < 5\%). DPIHP exhibited little effect on the polymerization process. With QTX as the photoinitiator, however, DPIHP played an essential role. Without DPIHP, all three QTX-CI systems failed to initiate polymerization (DC < 5\%). The QTX-DPIHP combination, on the other hand, was found to be a viable initiator system. The above results provide the critical information for the development of self-etching adhesive systems.
\end{abstract}

\section{Keywords}

self-etching adhesive; degree of conversion; photo-polymerization rate; acidic and hydrophilic monomer; FTIR

\section{INTRODUCTION}

Early-generation dentin adhesives are relative hydrophobic and usually cannot penetrate through the demineralized dentin layer. The development of the wet bonding technique ${ }^{1,2}$ has led to higher bond strength by preventing the collapse of demineralized dentin and

(C) 2008 Wiley Periodicals, Inc.

Correspondence to: Y. Wang; Wangyo@umkc.edu. 
enhancing the infiltration of adhesive into collagen networks. This requires the adhesives to be hydrophilic enough to wet the dentin and to be more moisture resistant. ${ }^{3}$ These adhesives usually use the total-etch technique, which involves separate acid etching and rinsing steps. ${ }^{4}$ However, excessive etching of dentin may produce weak bonding due to incomplete impregnation of the collagen fibrils by the resin at the base of the demineralized dentin. Furthermore, the amount of moisture in the demineralized dentin, which has a crucial effect on the bonding strength, cannot be clinically controlled, ${ }^{5-7}$ making the total-etch process extremely technique sensitive. The contemporary self-etching adhesives/primers were designed to combine both the etching function and the resin-forming function, thus eliminating rinsing steps. Compared to total-etch adhesives, self-etching adhesives are generally more user-friendly, less technique sensitive, and easier in achieving an acceptable seal. ${ }^{8,9}$

Although the bonding between self-etching adhesives and dentin ${ }^{10-20}$ has been extensively studied, most of such studies have so far focused on the structural and mechanical properties of the interface between the dentin and resulting resin. As to the polymerization process itself, little efforts have been devoted. As mentioned earlier, self-etching adhesives are relatively hydrophilic. Camphorquinone (CQ), the widely used photoinitiator, however, is relatively hydrophobic. It is known that $\mathrm{CQ}$ has difficulties in initializing polymerization of water-soluble monomers, such as HEMA in aqueous solutions. ${ }^{21}$ It is thus legitimate to ask how efficient CQ is in initializing the polymerization of relatively hydrophilic self-etching adhesives under wet conditions. Hayakawa et al. ${ }^{23-27}$ have employed QTX as a water soluble photoinitiator in hydrophilic dental adhesive formulations, and have shown that it can improve the bond strength of composite resin to dentin. Although these studies clearly point to the importance of water soluble initiators, how various initiator systems, whether being hydrophilic or hydrophobic, affect the polymerization rates and degree of conversion (DC) has not been explored. The objective of the present study was to gain deep understanding of how various initiators, coinitiators $(\mathrm{CI})$, and the additional third component found in model self-etching adhesives affect the photo-polymerization process. Our studied systems have CQ or QTX as the initiator, NPG, 4E or DMAEMA as the CI, DPIHP as the third component, and 2MP/HEMA as the monomers. The polymerization rates and DCs were evaluated via the FTIR/ATR spectroscopy. It is hypothesized that the different photoinitiator components would affect polymerization of acidic, aqueous 2MP/HEMAbased adhesive. In addition, because of its ability to enhance radical efficacy, addition of DPIHP will significantly increase both final conversion and overall conversion rate of all photoinitiator systems that do not contain it.

\section{MATERIALS AND METHODS}

\section{Model resin and photoinitiator systems}

The monomer mixtures used in this investigation consisted of 2MP (Aldrich, Milwaukee, WI) and HEMA (Acros Organics, Morris Plain, NJ) with a mass ratio of 1/1. This composition is similar to that used in commercial two-step self-etch dentin adhesive formulations such as Clearfil Liner Bond 2V (Kuraray America, Inc., New York, NY). The chemical structures of all compounds used in the initiator systems are shown in Figure 1. The following combinations of visible light CQ- and QTX-containing photoinitiator systems (all from Aldrich, Milwaukee, WI) were used: CQ/NPG, CQ/NPG/DPIHP, CQ/4E, CQ/4E/ DPIHP, CQ/DMAEMA, CQ/DMAEMA/DPIHP, CQ/DPIHP, QTX/NPG, QTX/NPG/ DPIHP, QTX/4E, QTX/4E/DPIHP, QTX/DMAEMA, QTX/DMAEMA/DPIHP, QTX/ DPIHP. The amount of CQ or QTX was $0.022 \mathrm{mmol}$ per gram of monomer. Equal molar CIs and/or DPIHP were used. 
When water was added to the above solutions, heavy water (deuterium oxide, $\mathrm{D}_{2} \mathrm{O}$ )

(Cambridge Isotope Laboratories, Inc., Andover, MA) was used. Using $\mathrm{D}_{2} \mathrm{O}$ instead of $\mathrm{H}_{2} \mathrm{O}$ was to avoid the interference of the IR absorption of $\mathrm{H}_{2} \mathrm{O}$ to that of $\mathrm{C}=\mathrm{C}$ double bonds in adhesives. $\mathrm{D}_{2} \mathrm{O}$ was added make either 5,15 , or $25 \%$ solutions. Shaking and sonication were required to yield well-mixed mixtures. All the chemicals in this study were used as received.

\section{Collecting real-time FTIR/ATR spectra}

Real-time in-situ monitoring of the photocuring of the different solutions was performed using an infrared spectrometer (Spectrum One, Fourier transform infrared spectrophotometer, Perkin-Elmer, Waltham, MA) at a resolution of $4 \mathrm{~cm}^{-1} .28,29$ The adhesive/water mixtures were cast on the diamond crystal top-plate of an attenuated total reflectance (ATR) accessory (Perkin-Elmer, Waltham, MA), covered with a mylar film. The ATR crystal was zinc selenide ( $\mathrm{ZnSe}$ ) with a transmission range between 650 and approximately $4000 \mathrm{~cm}^{-1}$. A time-based spectrum collector (Spectrum TimeBase, PerkinElmer) was used for continuous and automatic collection of spectra during polymerization at a rate of one spectrum every $0.4-0.6 \mathrm{~s}$. At this high rate and resolution, the signal-to-noise ratio of spectra is sufficient for spectral analysis. A 40-s exposure from a conventional dental light polymerization unit (Spectrum Light, Dentsply, Milford, DE) emitting $550 \mathrm{~mW} /$ $\mathrm{cm}^{2}$ was applied after 50 spectra had been recorded. Real-time IR spectra were continuously recorded for $300 \mathrm{~s}$ after light activation began. Three separate replications for each adhesive formulation were obtained. Data on the DC and polymerization rate were analyzed using analysis of variance (ANOVA), together with the T test $(p<0.05)$.

\section{Calculation of monomer conversion and polymerization rate}

The DC and polymerization rate were calculated for each adhesive formulation. The change of the band ratios profile with $1637 \mathrm{~cm}^{-1}(\mathrm{C}=\mathrm{C}) / 1715 \mathrm{~cm}^{-1}$ (carbonyl) was monitored and $\mathrm{DC}$ was calculated by the following equation

$$
\mathrm{DC}=\left(1-\frac{\text { Absorbance }_{1637 \mathrm{~cm}^{-1}}^{\text {sample }} / \text { Absorbance }_{1715 \mathrm{~cm}^{-1}}^{\text {sample }}}{\text { Absorbance }_{1637 \mathrm{~cm}^{-1}}^{\text {monomer }} / \text { Absorbance }_{1715 \mathrm{~cm}^{-1}}^{\text {monomer }}}\right) \times 100 \%
$$

which was based on the decrease in the absorption intensity band ratios during light curing. Two-point baseline and peak high ratio protocol were employed to measure the absorption intensity. The DC was calculated based on the last 20 spectra of time-resolved spectra. One DC value was an average of 60 points. The maximal polymerization rate $\left(R_{\max }\right)$ was determined using the maximum slope of linear region of the DC-time plots by using the least square linear fitting. The polymerization rate of each formulation was an average of three slopes.

\section{RESULTS}

Representative real-time FTIR spectra of one model adhesive formulation collected at different time during photopolymerization are shown in Figure 2. The band occurred at 1715 $\mathrm{cm}^{-1}$ is associated with carbonyl group in the resin monomers and the band at $1637 \mathrm{~cm}^{-1}$ is associated with $\mathrm{C}=\mathrm{C}$ in methacrylate monomers. It can be seen that the absorbance (peak height) of the $1715 \mathrm{~cm}^{-1}$ band remains unchanged while absorbance at $1637 \mathrm{~cm}^{-1}$ decreases indicating consumption of methacrylate $\mathrm{C}=\mathrm{C}$ bonds during polymerization. Based on these two bands, the DC at different curing time can be determined.

Time-based conversions of the model adhesives initiated by different CQ-containing initiator systems in the presence of 5, 15, and 25\% water are shown in Figure 3(a-c), 
respectively. Comparison of the plots in Figure 3 revealed different polymerization kinetics in the presence of the various CI systems. Most of the changes in DC were finished within $60 \mathrm{~s}$ after light activation. The results showed that for this very hydrophilic and acidic monomer mixture, CQ/DMAEMA could hardly initiate polymerization, whereas $4 \mathrm{E}$ was the best $\mathrm{CI}$ among the three. Adding the third component DPIHP did not result in any improvement on the initiating efficiency or polymerization of $\mathrm{CQ} / \mathrm{amine}$ systems.

Final conversion values for each formation in the presence of different water contents were calculated and are presented in Figure 4. Their polymerization rates determined based on the slopes of linear region of the plots are shown in Figure 5. The polymerization rates were not calculated for those systems with DC less than $10 \%$, due to the poor linear fitting. Among the three CQ-containing initiator systems, CQ/4E gave the highest $\mathrm{DC}(79.1 \pm 0.7 \%)$ and the fastest polymerization rate $(1.15 \pm 0.01)$ in the presence of $5 \%$ water. Addition of water had a dramatic effect on both the DC and rate of polymerization of systems initiated by this CQ/ $4 \mathrm{E}$ system $(p<0.05)$ : with increasing water content, both the rate and final conversion decreased (Figs. 4 and 5). However, for other initiator systems, addition of water did not seem to have such a dramatic effect. For all three CQ-containing systems, the incorporation of DPIHP did not cause any significant value changes $(p>0.05)$.

When QTX was used as the initiator, very different results were obtained. Time-based conversions of the model adhesives initiated by different QTX-containing initiator systems in the presence of 5, 15, and 25\% water are shown in Figure 6(a-c), respectively. The results showed that all three QTX-containing initiator/CI systems failed to start photopolymerization when DPIHP was not added. With DPIHP, the DC plateaus jumped from nearly zero $(0-5 \%)$ to the values in the range from $\sim 20 \%$ to $\sim 75 \%$ for the three systems at different water contents. In contrast to the CQ-containing systems, DPIHP played a very important role in QTX-containing initiator systems.

Final conversion values and polymerization rates for each QTX-containing formation in the presence of different water contents were calculated and are presented in Figures 7 and 8. Without DPIHP, the final DC values were all less than 5\%; the values for polymerization rate were not given due to poor fitting. Among all QTX-containing initiator systems, QTX/ 4E/DPIHP combination gave the best results: the DC at $15 \%$ water content $(73 \pm 1.7 \%)$ was almost twice as high as those of other two initiator systems. The highest polymerization rate was $2.13 \pm 0.01$ at $5 \%$ water content. With increasing water content, both the rate and final conversion decreased for all three DPIHP containing systems $(p<0.05)$, except for QTX/ 4E/DPIHP, in which we observed a slight increase in DC when water content increased from 5 to $15 \%$.

To find out if the amines are necessary for initiating polymerization, QTX and DPIHP, as well as CQ and DPIHP initiator systems were also studied. The relationships of DC via time of the model adhesives initiated by CQ/DPIHP, or QTX/DPIHP in the presence of 5, 15, and $25 \%$ water are shown in Figure 9. The conversion values and polymerization rates are presented in Figure 10. The results showed that QTX/DPIHP did initiate the polymerization of 2MP/HEMA mixture, while CQ/DPIHP did not. With DPIHP, QTX could initiate polymerization with or without the presence of a tertiary amine as the CI. Based on similarity between the value bar profiles in Figure 10 and those in Figures 7 and 8, the above results indicated that CIs did not play a critical role in the polymerization initiation process when QTX was employed as the initiator. 


\section{DISCUSSION}

It is well known that the bonding strength between the adhesive and dentin depends strongly on the degree of monomer conversion. Monomer conversion in a photo-initiated polymerization, on the other hand, is sensitive to the initiator and CI used, the types of monomers and the reaction medium. For self-etching model adhesives, the monomers are relatively acidic and hydrophilic. The polymerization is usually carried out in the presence of moisture. Although a number of self-etching adhesives have been well studied and commercially applied, no systematic studies have been carried out in regard to the understanding of how different initiators, CIs as well as other components affect the polymerization rate and monomer conversion. The results of this study support the first proposed hypothesis that the different photoinitiator system components dramatically affect polymerization of acidic, aqueous 2MP/HEMA-based resins. However, the results reject the second hypothesis. Addition of DPIHP had different effects on both final conversion and overall conversion rate of two different photoinitiator systems. With CQ as the photoinitiator, DPIHP exhibited little effect on the polymerization process. With QTX as the photoinitiator, however, DPIHP played an essential role. Without DPIHP, all three QTX containing systems failed to initiate polymerization.

There are numerous reported photoinitiator systems. Among these systems, the three CQcontaining initiators used in this study are very popular. It was shown that the reactivity ranking was $\mathrm{CQ} / 4 \mathrm{E}>\mathrm{CQ} / \mathrm{NPG}>\mathrm{CQ} / \mathrm{DMAEMA}$. Addition of the third component (iodonium salt) DPIHP did not cause any improvement on the kinetics of polymerization (Figs. 3 and 4). These results are different from the previous results on polymerization of HEMA or HEMA/BisGMA model resin initiated by these systems. It was reported that ${ }^{21,22}$ in $\mathrm{CQ} / \mathrm{amine}$ initiator system, the incorporation of iodonium salt could facilitate the polymerization process of HEMA. We have previously shown the iodonium salt DPIHP could also accelerate the polymerization of the BisGMA/HEMA mixture. ${ }^{30}$ For example, the CQ/DMAEMA/DPIHP initiator system ${ }^{30}$ was shown to be an excellent one in initiating the BisGMA/HEMA (weight ratio 60/40) model adhesives with very high DC and polymerization rate in the presence of water. The same system, however, could hardly initiate the 2MP/HEMA (weight ratio 1/1) model adhesive (DC < 5\%) (Fig. 4).

To explain the above results, the initiating process of a CQ-amine-iodonium salt is discussed below, which is generally accepted as the following: ${ }^{22}$
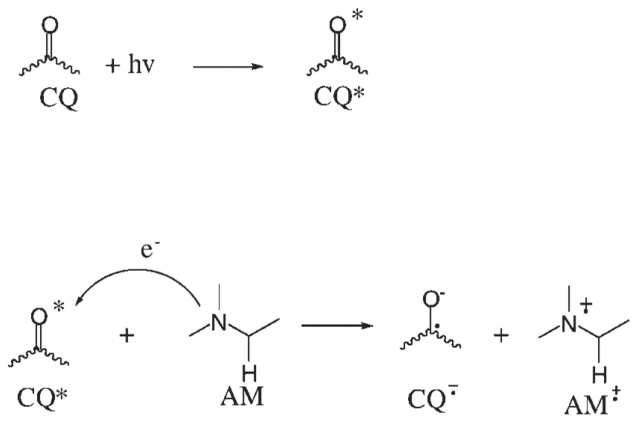


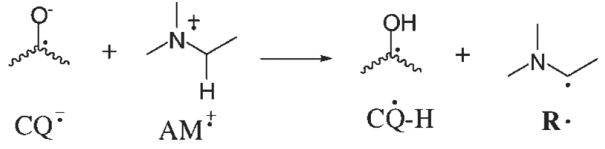

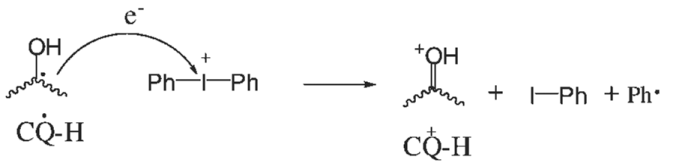<smiles>CC(C)(C)C(=O)[PH2+]</smiles>

For the model adhesives employed in this study, our results indicated that the amines applied played a critical role in initiating polymerization. Among the three amines used, 4E gave the highest DC whereas DMAEMA the lowest. This trend is consistent with the basicity order of the three amines. 4E is the weakest base whereas DMAEMA is the strongest one. Apparently, the higher acidity of the monomer impeded the polymerization, presumably by forming an ammonium salt with DMAEMA thus preventing DMAEMA from serving as an electron donor. The stronger the basicity of the amine is, the higher the tendency of salt formation between the amine and the acidic monomer will be. Salt formation blocks the electron transfer step [Eq. (2)], thus preventing radical initiation.

Although DPIHP was found to be a good promoter for CQ-amine photoinitiated polymerization of HEMA and bisGMA/HEMA systems, the addition of DPIHP did not result in significant improvement in DCs for all three CQ-based initiator systems. Clearly, this has something to do with the acidity of monomer 2MP. The acidity of 2MP limits the regeneration of CQ [Eq. (5)] which likely accounts for the inconsequential effect of DPIHP for acidic monomers. These results indicate that when selecting a photoinitiator system, one must take into account the acidity and the hydrophilicity of the monomers as those properties affect strongly the polymer initiation process.

Although DPIHP appears to be ineffective for acidic monomers using CQ-amine as the initiator-coinitiator, its presence is essential when QTX is used as the initiator. Without DPIHP, no or negligible polymerization was observed. With DPIHP, QTX can initiate polymerization with or without the added amines, although the addition of amines

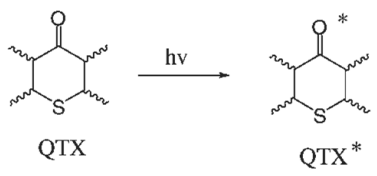



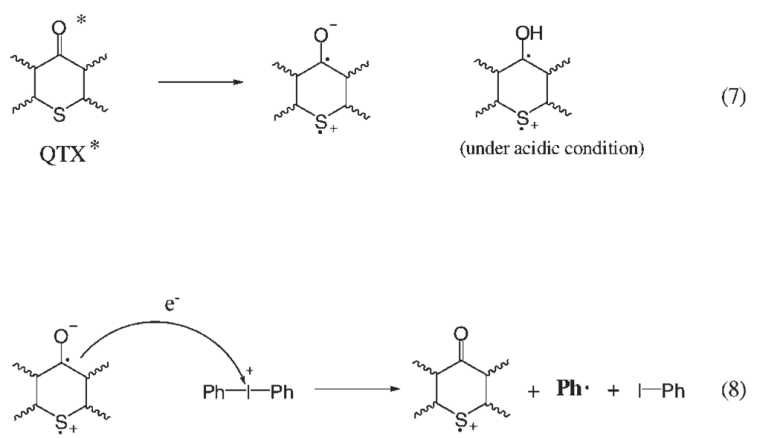

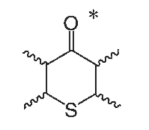

QTX*

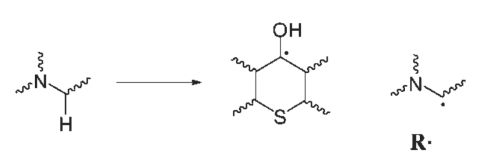

(9)

improves the DCs to some extent. These results indicate that photo-excited QTX can react with DPIHP to generate active radicals which initiate polymerization.

To study the acidity effect on the polymerization, 2MP was replaced with equal amount of HEMA in the formulation with 5\% water. The DCs of QTX-DPIHP and QTX-4E initiator systems were $7 \pm 1 \%$ and $7.9 \pm 0.8 \%$, respectively. With 2MP, the DC of QTX-4E initiator system decreased to $3 \pm 1 \%$ whereas that of QTX-DPIHP increased $60 \pm 1 \%$. These results may be explained by a proposed initiation mechanism shown in Eqs. (6)-(9). After QTX is excited by a photon [Eq. (6)], it can undergo either an intramolecular charge transfer (CT) [Eq. (7)] or intermolecular CT [Eq. (9)] process. For the QTX-4E system, intermolecular CT process, followed by $\mathrm{H}$-abstraction results in active alkyl radicals which initiator polymerization. For the QTX-DPIHP system, active phenyl radical is generated after consecutive intramolecular [Eq. (7)] and intermolecular [Eq. (8)] CT processes. Both QTX-4E and QTX-DPIHP can thus initiate polymerization. They both, however, are not efficient initiator systems for nonacidic monomers. Under acidic condition, intramolecular $\mathrm{CT}$ process is significantly enhanced due to the neutralization of the oxide anion with the proton to form hydroxyl group [see Eq. (7)]. For the QTX-DPIHP system, this enhanced intramolecular CT process results in higher concentration of the phenyl radical (after step 8), and thus improves the polymerization process. For the QTX-4E system, however, the enhanced intramolecular $\mathrm{CT}$ process leads to less competitive intermolecular $\mathrm{CT}$ process and therefore lower concentration of active alkyl radicals. It is thus understandable why acidic monomers polymerize well with QTX-DPIHP as the initiator but poorly with QTX-4E as the initiator. As stated earlier, incorporation of 4E into the QTX-DPIHP system enhances the polymerization rate and DC. This can be explained by the fact that both the intramolecular and intermolecular CT processes lead to active radicals.

In summary, two different initiator systems have been studied for the photopolymerization of 2MP/HEMA model adhesives. For CQ-containing initiator systems, amine as the coinitiator plays a critical role in the initiating process, while DPIHP as an added third component does not lead to noticeable improvement in the polymerization. The polymerization performance depends strongly on the amines applied. Among the three amines studied, 4E gives the best results whereas DMAEMA can hardly initiate polymerization. These results are opposite to their relative basicity with the weakest base

J Biomed Mater Res A. Author manuscript; available in PMC 2013 June 05. 
(4E) showing the best performance. Such data indicate that protonation of the amines is a major obstacle preventing the polymerization of acidic monomers. For QTX systems, QTXDPIHP alone can effectively initiate the polymerization of acidic monomers. This is explained by the existence of dominant intramolecular CT process associated with the excited QTX molecule. It also accounts for the observation that QTX-amine alone could not initiate the polymerization of acidic monomers.

\section{Acknowledgments}

Contract grant sponsor: National Institute of Dental and Craniofacial Research, Bethesda, MD; contract grant numbers: R01DE14392, K25DE015281

\section{References}

1. Kanca J. Improved bond strength through acid etching of dentin and bonding to wet dentin surfaces. J Am Dent Assoc. 1992; 123:235-243.

2. Gwinnett AJ. Dentin bond strength after air drying and rewetting. Am J Dent. 1994; 7:144-148. [PubMed: 7993603]

3. Fusayama T, Nakamura M, Kurosaki N, Iwaku M. Non-presure adhesion of a new adhesive restoration resin. J Biomed Mater Res. 2005; 58:1364-1370.

4. Fusayama T, Nakamura M, Kurosaki N, Iwaku M. Non-pressure adhesion of a new adhesive restorative resin. J Dent Res. 1979; 58:1364-1370. [PubMed: 372267]

5. Tay FR, Gwinnett AJ, Wei SHY. An optical, micromorphological study of surface moisture in the acid-conditioned, resin-dentin interface. Am J Dent. 1996; 9:43-48. [PubMed: 9002816]

6. Tay FR, Gwinnett AJ, Wei SHY. The overwet phenomenon: A scanning electron microscopic study of surface moisture in the acid-conditioned, resin-dentin interface. Am J Dent. 1996; 9:109-114. [PubMed: 9002800]

7. Tay FR, Gwinnett AJ, Wei SHY. The overwet phenomenon: A transmission electron microscopic study of surface moisture in the acid-conditioned, resin-dentin interface. Am J Dent. 1996; 9:161166. [PubMed: 9002792]

8. Wananabe I, Nikaido T, Nakabayashi N. Effect of adhesion promoting monomers on adhesion to ground dentin. J Jpn Soc Dent Mater Dev. 1990; 9:888-893.

9. Kiyomura M. Bonding strength to bovine dentin with 4-META/MMA-TBB resin: Long-term stability and influence of water. Dent Mater J. 1987; 6:860-872.

10. Watanabe I, Nakabayashi N, Pashley DH. Bonding to ground dentin by a Phenyl-P self-etching primer. J Dent Res. 1994; 73:1212-1220. [PubMed: 8046111]

11. Yoshiyama M, Carvalho RM, Sano H, Horner JA, Brewer PD, Pashley DH. Regional bond strengths of resins to human root dentin. J Dent. 1996; 24:435-442. [PubMed: 8990690]

12. Prati C, Chersoni S, Mongiorgi R, Pashley DH. Resin-infiltrated dentin layer formation of new bonding systems. Oper Dent. 1998; 23:185-194. [PubMed: 9760921]

13. Yoshiyama M, Matsuo T, Ebisu S, Pashley DH. Regional bond strength of self-etching/selfpriming adhesive systems. J Dent. 1998; 26:609-616. [PubMed: 9754751]

14. Aksornmuang J, Nakajima M, Foxton RM, Tagami J. Effect of prolonged photo-irradiation time of three self-etch systems on the bonding to root canal dentin. J Dent. 2006; 34:389-397. [PubMed: 16236418]

15. Miyasaka K, Nakabayashi N. Combination of EDTA conditioner and phenyl-P/HEMA self-etching primer for bonding to dentin. Dent Mater. 1999; 15:153-157. [PubMed: 10551077]

16. Tay FR, Pashley DH. Aggressiveness of contemporary self-etching systems. I. Depth of penetration beyond dentin smear layers. Dent Mater. 2001; 17:296-308. [PubMed: 11356206]

17. Tay FR, Pashley DH. Aggressiveness of contemporary self-etching systems. II. Etching effects on unground enamel. Dent Mater. 2001; 17:430-444. [PubMed: 11445211]

18. Oliveira SS, Marshall SJ, Hilton JF, Marshall GW. Etching kinetics of a self-etching primer. Biomaterials. 2002; 23:4105-4112. [PubMed: 12182312] 
19. Wang Y, Spencer P. Continuing etching of an all-in-one adhesive in wet dentin tubules. J Dent Res. 2005; 84:350-354. [PubMed: 15790742]

20. De la Macorra JC, San-Nicolas A. Method to compare $\mu$-tensile bond strength of a self-etching adhesive and $\mu$-cohesive strength of adjacent dentin. Dent Mater. 2005; 21:946-953. [PubMed: 15878782]

21. Wang Y, Spencer P, Yao X, Ye Q. Effect of coinitiator and water on the photoreactivity and photopolymerization of HEMA/camphoquinone-based reactant mixtures. J Biomed Mater Res A. 2006; 78:721-728. [PubMed: 16739171]

22. Padon KS, Scranton AB. A mechanistic investigation of a three-component radical photoinitiator system comprising methylene blue, N-methyldiethanolamine, and Diphenyliodonium chloride. J Polym Sci Part A: Polym Chem. 2000; 38:2057-2066.

23. Hayakawa T, Horie K. Effect of water-soluble photoinitiator on the adhesion between composite and tooth substrate. Dent Mater. 1992; 8:351-353. [PubMed: 1303380]

24. Hayakawa T, Nemoto K, Horie K. Adhesion of composite to polished dentin retaining its smear layer. Dent Mater. 1995; 11:218-222. [PubMed: 8600016]

25. Nakanuma K, Hayakawa T, Tomita T, Yamazaki M. Effect of the application of dentin primers and a dentin bonding agent on the adhesion between the resin-modified glass-ionomer cement and dentin. Dent Mater. 1998; 14:281-286. [PubMed: 10379257]

26. Hayakawa T, Kikutake-Sugiyama K, Fukushima T, Nemoto K. Development of self-etching primer adhesive in all-in-one bonding system. Dent Mater J. 2005; 24:251-256. [PubMed: 16022447]

27. Hayakawa T, Kikutake-Sugiyama K, Nemoto K. Efficacy of water-soluble photoinitiator on the adhesion of composite resin to bovine teeth in all-in-one bonding system. Dent Mater J. 2005; 24:213-218. [PubMed: 16022441]

28. Ye Q, Wang Y, Williams K, Spencer P. Characterization of photopolymerization of dentin adhesives as a function of light source and irradiance. J Biomed Mater Res Part B: Appl Biomater. 2006; 80:440-446. [PubMed: 16850459]

29. Ye Q, Spencer P, Wang Y, Misra A. Relationship of solvent to the photopolymerization process, properties, and structure in model dentin adhesives. J Biomed Mater Res Part A. 2007; 80:342350.

30. Guo XL, Wang Y, Spencer P, Ye Q, Yao XM. Effects of initiator and water on photopolymerization of BisGMA/HEMA-based model adhesives by time-resolved FTIR analysis. Dent Mater. 2008; 24:824-831. [PubMed: 18045679] 


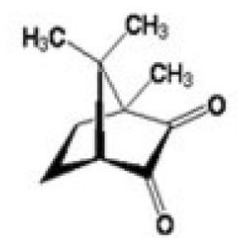

$\mathrm{CQ}$<smiles>CCOC(=O)c1ccc(N(C)C)cc1</smiles>

$4 \mathrm{E}$<smiles>C=C(C)C(=O)OCCN(C)C</smiles>

DMAEMA<smiles></smiles>

QTX<smiles>O=C(O)CNc1ccccc1</smiles>

NPG<smiles>[PH3+][PbH2]c1ccccc1</smiles>

DPIHP

Figure 1.

Chemical structures of components used in the initiator systems. camphorquinone (CQ); [3(3,4-dimethyl-9-oxo-9H-thioxanthen-2-yloxy)-2-hydroxylpropy] trimethylammonium chloride (QTX); ethyl 4-dimethylaminobenzoate (4E); n-phenylglycine (NPG); 2(dimethylamino) ethyl methacrylate (DMAEMA); diphenyliodonium hexafluorophosphate (DPIHP). 


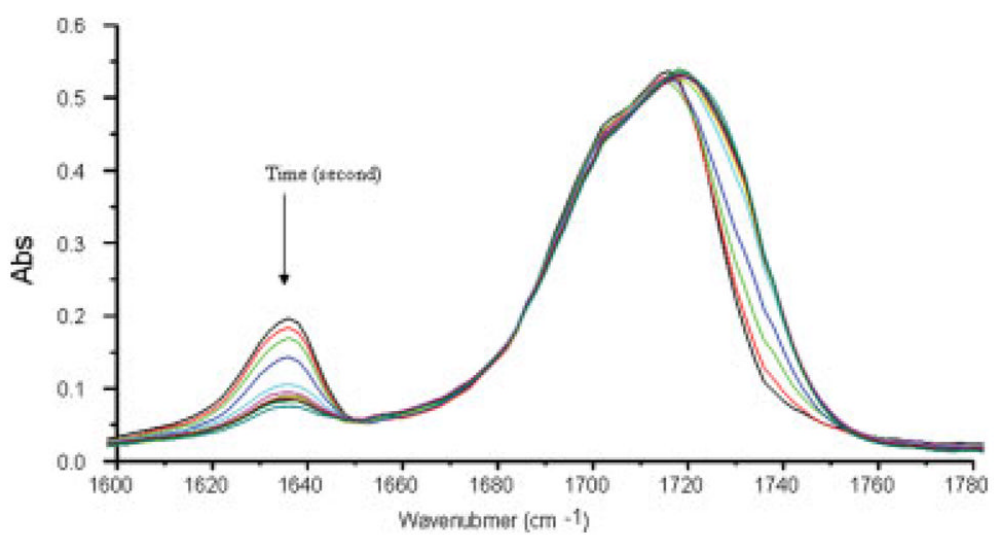

Figure 2.

Representative FTIR spectra of the 2MP/HEMA model adhesive containing CQ/4E initiator system in the presence of 5\% water collected at different time (from $30 \mathrm{~s}$ to $140 \mathrm{~s}$, every 10 s). [Color figure can be viewed in the online issue, which is available at www.interscience.wiley.com.] 

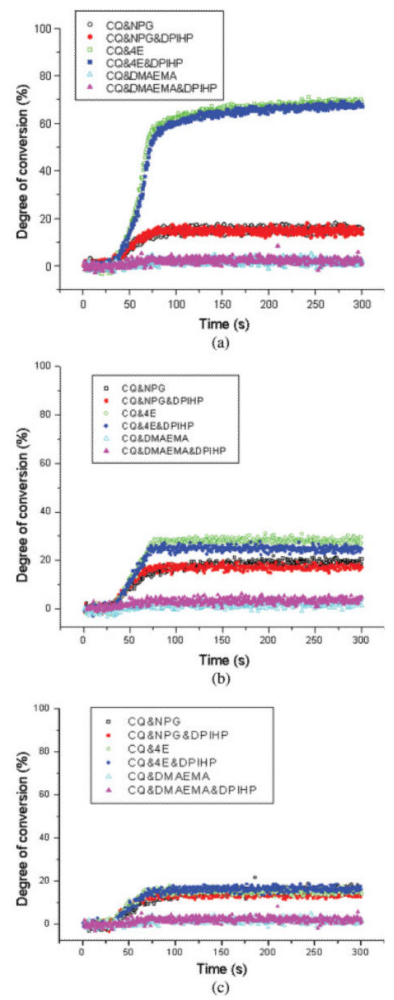

Figure 3.

Real-time conversion plots of the 2MP/HEMA model adhesives initiated by different CQcontaining initiator systems in the presence of $5 \%$ (a), $15 \%$ (b), and $25 \%$ (c) water. Curing light exposure duration was $40 \mathrm{~s}$. [Color figure can be viewed in the online issue, which is available at www.interscience.wiley.com.] 


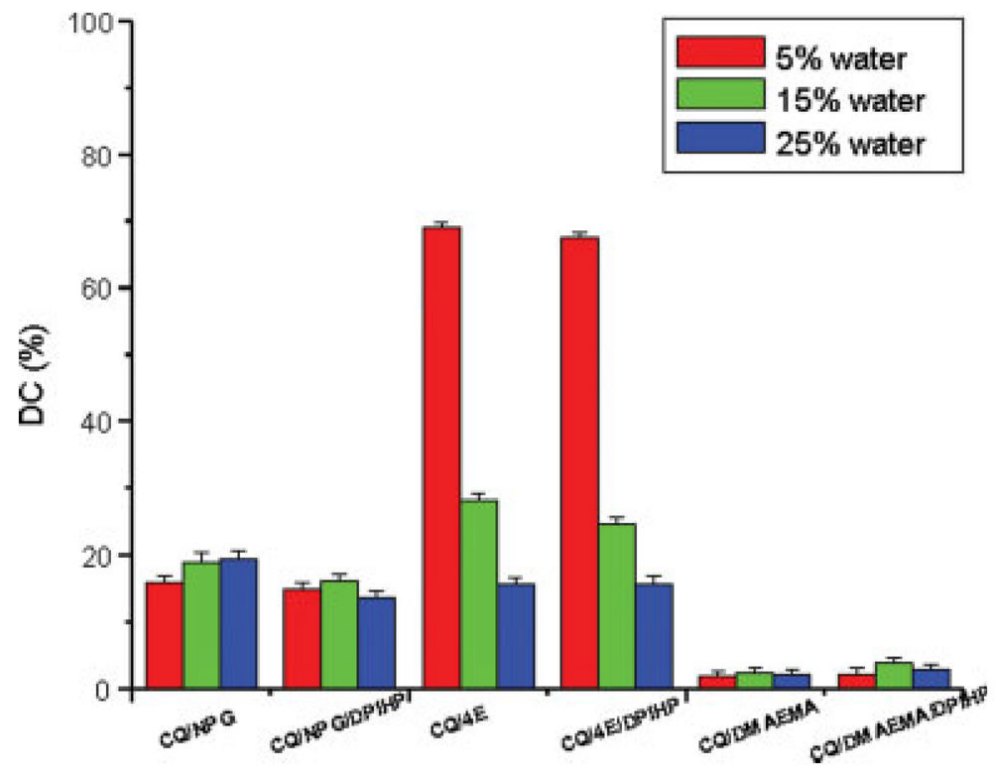

Figure 4.

The degree of conversion of the model adhesives initiated by different CQ-containing initiator systems in the presence of different water content. [Color figure can be viewed in the online issue, which is available at www.interscience.wiley.com.] 


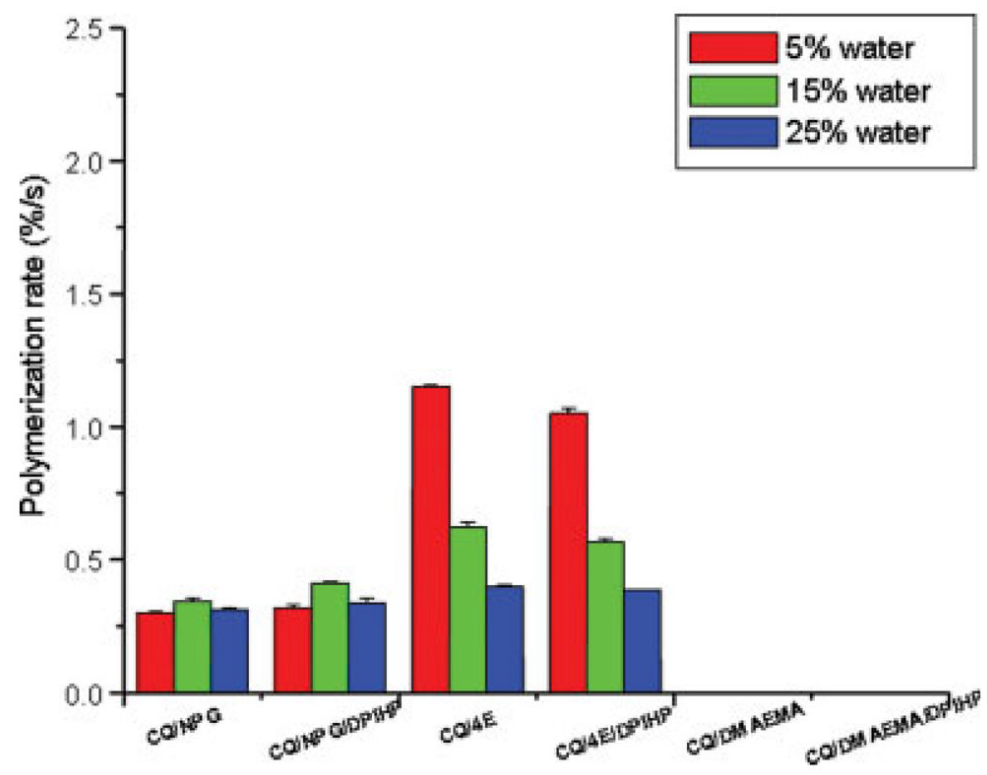

Figure 5.

Polymerization rates of the model adhesives initiated by different CQ-containing initiator systems in the presence of different water content. [Color figure can be viewed in the online issue, which is available at www.interscience.wiley.com.] 

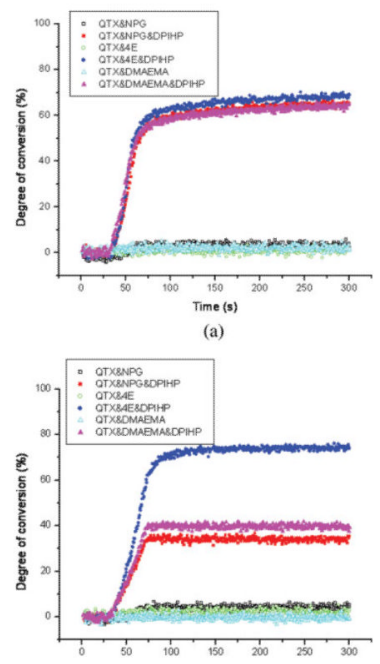

(b)

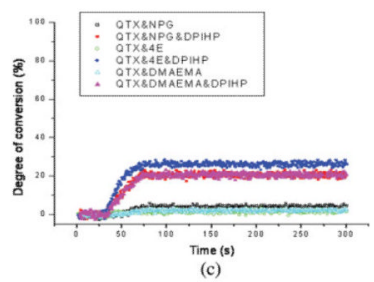

Figure 6.

Real-time conversion plots of the 2MP/HEMA model adhesives initiated by different QTXcontaining initiator systems in the presence of $5 \%$ (a), $15 \%$ (b), and $25 \%$ (c) water. Curing light exposure duration was $40 \mathrm{~s}$. [Color figure can be viewed in the online issue, which is available at www.interscience.wiley.com.] 


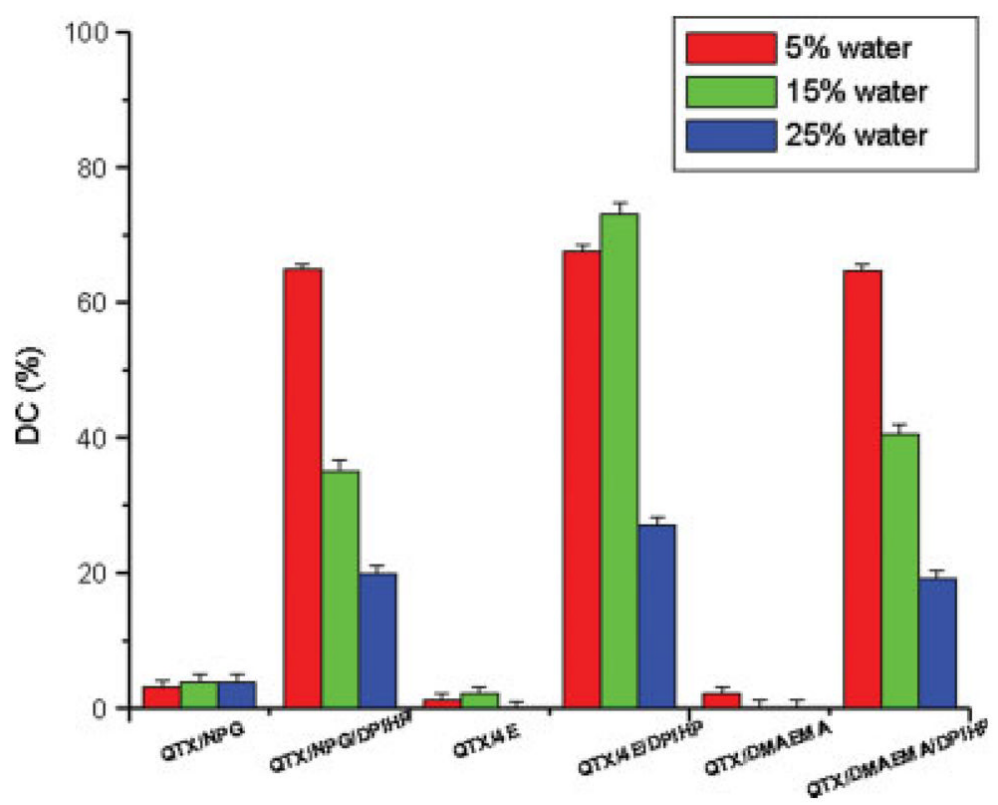

Figure 7.

The degree of conversion of the model adhesives initiated by different QTX-containing initiator systems in the presence of different water content. [Color figure can be viewed in the online issue, which is available at www.interscience.wiley.com.] 


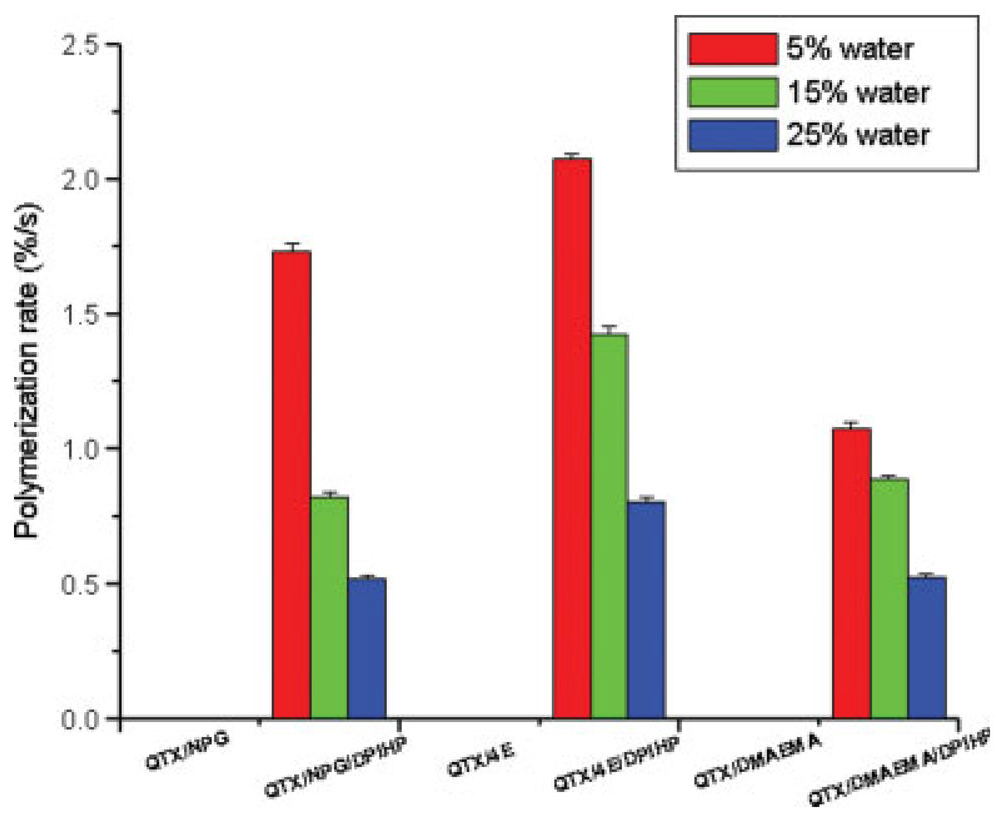

Figure 8.

Polymerization rates of the model adhesives initiated by different QTX-containing initiator systems in the presence of different water content. [Color figure can be viewed in the online issue, which is available at www.interscience.wiley.com.] 


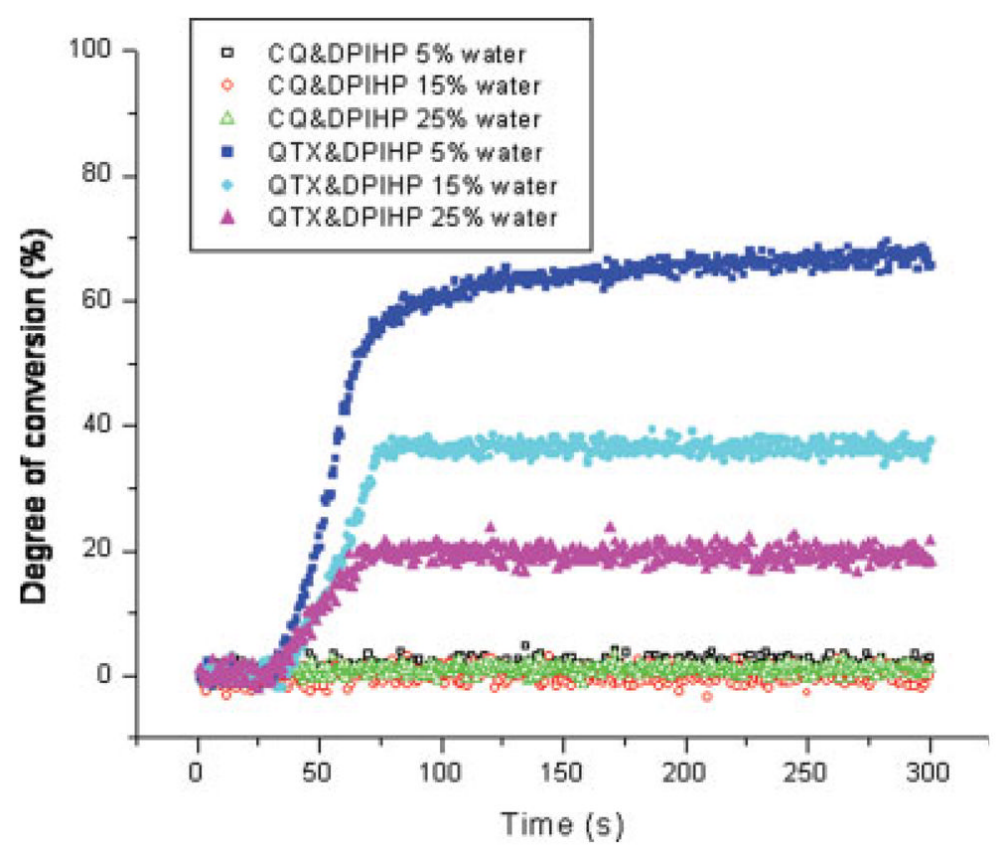

Figure 9.

Real-time conversion plots of the 2MP/HEMA model adhesives initiated by different amine free initiator systems in the presence of $5 \%$ (a), $15 \%$ (b), 25\% (c) water. Curing light exposure duration was $40 \mathrm{~s}$. [Color figure can be viewed in the online issue, which is available at www.interscience.wiley.com.] 

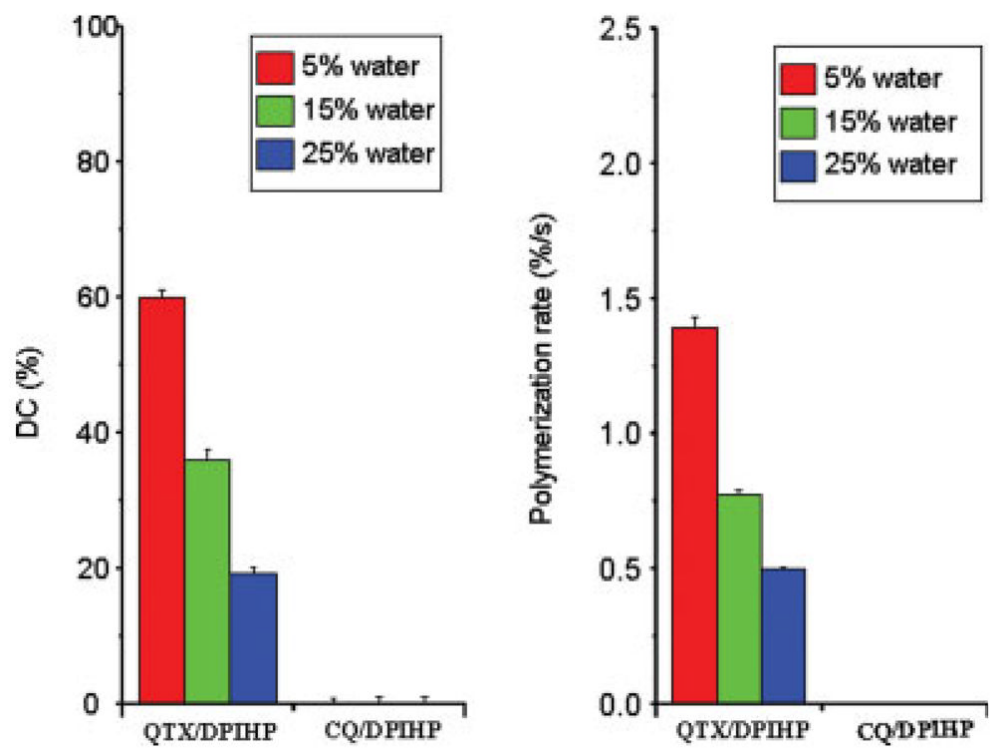

Figure 10.

The degrees of conversion and polymerization rates of the model adhesives initiated by different amine-free initiator systems in the presence of different water content. [Color figure can be viewed in the online issue, which is available at www.interscience.wiley.com.] 